\title{
USO DE TABACO E PERFIL LIPÍDICO-LIPOPROTÉICO PLASMÁTICO EM ADOLESCENTES
}

\author{
Dartagnan Pinto Guedes*, Joana Elisabete Ribeiro Pinto Guedes, Décio Sabbatini Barbosa, Jair Aparecido de Oliveira
}

Trabalho realizado na Universidade Estadual de Londrina, Paraná

\author{
*Correspondência: \\ Rua Ildefonso Werner, 177 \\ Condomínio Royal Golf \\ Londrina/PR \\ Cep: 86055-545 \\ darta@sercomtel.com.br
}

\begin{abstract}
RESUMO
Oвjetivo. Analisar o impacto quanto ao uso de tabaco no perfil lipídico-lipoprotéico plasmático em amostra representativa de adolescentes.
\end{abstract}

Métodos. A amostra foi constituída por 452 sujeitos (246 moças e 206 rapazes) com idades entre 15 e 18 anos. Os participantes completaram questionário estruturado auto-administrado com relação ao uso de tabaco. As concentrações de lipídeos-lipoproteínas plasmáticas foram estabelecidas mediante procedimentos laboratoriais. Os procedimentos da análise de covariância, controlando a participação da ingestão de gordura saturada e de colesterol dietético, foram empregados para identificar as diferenças entre os valores médios. As estimativas de odds ratio foram utilizadas para estabelecer o risco relativo dos adolescentes fumantes apresentarem perfil lipídico-lipoprotéico de risco aterogênico.

Resultados. A proporção de fumantes foi de $20,9 \%$ entre os rapazes e 15,4\% entre as moças. 0 consumo médio de cigarros por dia foi de 9,2 $\pm 4,7$ nos rapazes e 5,6 $\pm 3,1$ nas moças. Quando comparados com não fumantes, rapazes e moças fumantes apresentaram níveis séricos de colesterol total, LDL-colesterol, trigglicerídeos e apolipoproteína BI 00 significativamente mais elevados, e níveis séricos de HDL-colesterol significativamente menores. Adolescentes fumantes tenderam a demonstrar risco de níveis de lipídeos-lipoproteinas plasmáticas alterados duas vezes maior que não fumantes.

Conclusã̃o. Intervenções direcionadas à adoção de um estilo de vida saudável, incluindo abstenção do uso de tabaco, deverão iniciar-se em idades precoces na tentativa de prevenir ou retardar o desenvolvimento de lesões ateroscleróticas e minimizar o aparecimento de coronariopatias prematuras na idade adulta.

Unitermos: Fumo. Apolipoproteína. Colesterol. Adolescência.

\section{INTRODUÇÃO}

Apesar de seus efeitos deletérios no estado de saúde serem amplamente difundidos e conhecidos, o uso de tabaco constitui uma das principais causas previsíveis de morbi-mortalidade ${ }^{1-8}$. Atualmente, em todo o mundo, estima-se que 4 milhões de mortes prematuras/ano sejam atribuídas às doenças e às complicações associadas ao uso de tabaco, sendo que $70 \%$ dessas mortes ocorrem em países subdesenvolvidos ou em processo de desenvolvimento?

Informações epidemiológicas mostram que a experimentação do tabaco vem ocorrendo cada vez mais precocemente. Estudos apontam que por volta de $90 \%$ dos adultos fumantes iniciaram o uso de tabaco antes dos 18 anos de idade ${ }^{10}$. Desse modo, considerando a tendência crescente quanto à aquisição do hábito de fumar entre os jovens, é projetado um aumento próximo de 8,4 milhões de mortes prematuras/ano em 2020 ocasionadas direta ou indiretamente pelo uso de tabaco"l.

Levantamentos realizados em diferentes países sugerem que, embora possa haver ampla variação de acordo com o sexo, o grupo etário e o país considerado, a prevalência de adolescentes fumantes em todo o mundo se aproxima de I5\% ${ }^{12,13}$. No Brasil, dados representativos de 27 capitais indicam que por volta de 10\% dos escolares brasileiros fazem uso de tabaco ${ }^{14}$.

Apesar da inalaçãao de substâncias tóxicas produzidas pelo fumo estar estreitamente associada ao aparecimento e ao desenvolvimento de alguns tipos de câncer e à manifestação de graves distúrbios respiratórios ${ }^{15-18}$, as doenças cardiovasculares são as mais importantes consequêencias do uso de tabaco 3-7,19,20. Importantes alterações em mecanismos funcionais e metabólicos têm sido descritas para explicar os efeitos nocivos do tabaco no sistema cardiovascular, entre as quais modificações nas concentrações de lipídeos-lipoproteinas plasmáticas vêm sendo bem documentadas. Quando comparados aos não fumantes, os adultos fumantes tendem a apresentar um perfil plasmático aterogênico mais comprometido ${ }^{4-6}$.

No entanto, poucos são os estudos publicados que procuram reportar o impacto quanto ao uso de tabaco no perfil lipídicolipoprotéico plasmático de sujeitos ainda em idades jovens. Sendo assim, o objetivo do presente estudo foi analisar essas variáveis em amostra representativa de adolescentes e, desse modo, oferecer informações relacionadas à extensão a que o uso de tabaco possa estar associado no que diz respeito às modificações adversas nas concentrações de lipídeos-lipoproteinas plasmáticas na população jovem.

\section{MÉtodos}

Para elaboração do estudo foram utilizadas informações contidas no banco de dados construído a partir do projeto de pesquisa Atividade Física, Composição da Dieta e Fatores de Risco Predisponentes às Doenças Cardiovasculares em Adolescentes, que inclui adolescentes entre 15 e 18 anos de idade de ambos os sexos.

O projeto de pesquisa teve como alvo escolares regularmente matriculados no ensino médio do Colégio de Aplicação ligado à 
Universidade Estadual de Londrina, PR. Optou-se por envolver sujeitos que freqüentavam unicamente essa escola, por conta das características longitudinais do estudo (experimentação de programas de educação para a saúde mediante intervenções dietéticas e de prática de exercícios físicos), e por sua representatividade no universo de escolares de ensino médio do município.

Protocolos de intervenção no estudo foram aprovados pelo Comitê de Ética em Pesquisa da Universidade Estadual de Londrina e acompanham normas da Resolução 196/96 do Conselho Nacional de Saúde sobre pesquisa envolvendo seres humanos. Inclusão dos sujeitos no estudo ocorreu por desejo de participar do experimento e mediante autorização dos pais ou responsáveis. Para tanto, todos escolares matriculados no ano letivo de 2003, juntamente com seus pais ou responsáveis, foram contatados e informados quanto à natureza e aos objetivos do estudo. Dos 518 escolares matriculados, 452 (246 moças e 206 rapazes) concordaram em participar do estudo.

Como informaçãa adicional da amostra analisada no estudo, destacase que, com base nos critérios de classificação socioeconômica das famílias dos escolares, mediante informações quanto ao nível de escolaridade do chefe da família, às condições de moradia, à posse de utensílios domésticos, automóveis e número de empregados domésticos ${ }^{21}$, observou-se que $22 \%$ dos escolares foram categorizados em menor nível socioeconômico, 27\% em maior nível e 51\% em níveis intermediários. Com relação aos critérios de classificação de maturação sexual sugeridos por Tanner ${ }^{22}, 10 \%$ das moças analisadas apresentavam desenvolvimento mamário equivalente ao estágio III, $61 \%$ ao estágio IV e 29\% ao estágio V. Entre os rapazes, $49 \%$ se encontravam no estágio IV de desenvolvimento de pilosidade pubiana, e os $51 \%$ restantes no estágio $V$.

As dosagens dos lipídeos-lipoproteínas plasmáticas foram realizadas mediante coleta de amostras de sangue venoso na prega do cotovelo, após período de I Oha I 2 h em jejum, entre $7 \mathrm{~h}$ e $8 \mathrm{~h}$ da manhã. O soro foi imediatamente separado por centrifugação, sendo determinados teores de triglicerídeos (TG), colesterol total (CT) e as frações, lipoproteínas de baixa densidade (LDL-C) e de alta densidade (HDL-C), apolipoproteínas AI (ApoA) e BI 00 (ApoB). Determinou-se - CT pelo método enzimático colesterol oxidase/peroxidase em aparelho espectofotômetro. O HDL-C foi medido pelo método reativo precipitante, e o LDL-C calculado pela fórmula de Friedewald ${ }^{23}$. Os TG foram determinados pelo método enzimático glicerol. As ApoA e ApoB foram dosadas pelo método imunoturbidimétrico, utilizando autoanalisador bioquímico Cobas Mira Plus ${ }^{\circledR}$. Além dos valores absolutos de cada componente plasmático, consideraram-se as relações CT/HDL-C, LDL-C/HDL-C e ApoB/ApoA. Os valores de referência empregados para definir um perfil lipídico-lipoprotéico de risco aterogênico acompanharam proposta apresentada para adolescentes no Consenso Brasileiro sobre Dislipidemias ${ }^{24}$.

O hábito quanto ao uso de tabaco foi identificado mediante análise das respostas apresentadas pelos adolescentes a questionário auto-administrado, padronizado, pré-codificado e anônimo, previamente testado, elaborado especificamente para esta finalidade. Considerou-se como fumantes aqueles adolescentes que responderam fumar pelo menos uma vez por semana ou diariamente no último mês e como ex-fumantes aqueles que, na ocasião da aplicação do questionário, não eram fumantes, mas que o haviam sido no passado.
Na tentativa de controlar eventuais efeitos de confusão no delineamento do estudo, foram consideradas informações dietéticas às relacionadas à ingestão de gordura saturada e de colesterol. Indicadores associados à prática habitual de atividade física não foram considerados, tendo em vista achados publicados anteriormente não terem apontado, nesta mesma casuística, influência significativa do sedentarismo nas concentrações de lipídeos-lipoproteínas plasmáticas consideradas de risco aterogênico ${ }^{25}$.

As informações acerca da ingestão de gordura saturada e de colesterol foram obtidas a partir de registros dietéticos em quatro dias da mesma semana, mediante utilização do software Sistema de Apoio à Decisão em Nutrição - Versão 2.5, idealizado pelo Centro de Informática em Saúde da Escola Paulista de Medicina/Universidade Federal de São Paulo ${ }^{26}$. Para análise do componente dietético, foram calculados os escores da RISCC (razão entre a ingestão de gordura saturada e de colesterol e o consumo calórico/dia) pela fórmula [I,0I(ingestão de gordura saturada em gramas) + 0,05 (ingestão de colesterol em mg)] / (consumo calórico em $\mathrm{kcal} / \mathrm{l} 000$ ).

O tratamento estatístico das informações foi realizado mediante 0 pacote computadorizado Statistical Package for the Social Science (SPSS), versão 13.0. Para análise das variáveis contínuas recorreu-se aos procedimentos da estatística descritiva. As comparações entre os valores equivalentes aos lipídeos-lipoproteínas plasmáticas dos adolescentes de ambos os sexos fumantes e não fumantes foram realizadas mediante análise de covariância. Para o controle dos efeitos adicionais associados aos componentes dietéticos, os escores da RISCC foram considerados como covariáveis na análise estatística.

Os valores de odds ratio (OR), estabelecidos por intermédio da análise de regressão logística binária, também controlando os escores da RISCC, foram utilizados para estabelecer estimativas quanto ao risco relativo dos adolescentes fumantes apresentarem perfil lipídico-lipoprotéico de risco aterogênico. Adotou-se intervalo de confiança de 95\%.

\section{Resultados}

Na época em que os dados do estudo foram coletados, 15,4\% das moças e $20,9 \%$ dos rapazes admitiram ser fumantes. Com relação à idade em que iniciaram o uso de tabaco, $15 \%$ dos fumantes relataram ter experimentado cigarro pela primeira vez antes dos 14 anos de idade. Para as idades de 14,15 e 16 anos, foram observadas proporções próximas de $31 \%, 26 \%$ e 25\%, respectivamente. Somente 3\% dos adolescentes que admitiram ser fumantes afirmaram ter iniciado a sua prática depois dos 16 anos. Em média, a quantidade de cigarros consumidos por dia foi mais elevada entre os rapazes $(9,2 \pm 4,7)$ do que entre as moças $(5,6 \pm 3,1)$.

Para análise do impacto quanto ao uso de tabaco no perfil lipídico-lipoprotéico plasmático, os adolescentes reunidos no estudo foram divididos em dois grupos: fumantes e não fumantes. Em razão de sua menor representatividade $(2,4 \%$ das moças e $3,4 \%$ dos rapazes), os ex-fumantes não foram considerados no estudo. Os escores médios relacionados à RISCC apresentaram dimensões entre 14,2 \pm 0,4 (moças não fumantes) e 18,9 \pm 0,7 (rapazes fumantes), não demonstrando diferenças significativas entre os adolescentes fumantes e não fumantes. 
Tabela I - Informações estatísticas equivalentes à análise de covariância, controlando as informações associadas à ingestão de gordura saturada e colesterol dietético, quanto às concentrações de lipídeos-lipoproteínas plasmáticas de adolescentes fumantes e não fumantes

\begin{tabular}{|c|c|c|c|c|}
\hline & \multicolumn{2}{|c|}{ Moças } & \multicolumn{2}{|c|}{ Rapazes } \\
\hline & Fumantes & Nãofumantes & Fumantes & Nãofumantes \\
\hline $\mathrm{CT}(\mathrm{mg} / \mathrm{dl})$ & $\overline{153,9 \pm 21,7}$ & $\mid 40,2 \pm 22,7^{\mathrm{a}}$ & $\overline{138,2 \pm 22,0}$ & $128,3 \pm 23,3^{a}$ \\
\hline $\mathrm{HDL}-\mathrm{C}(\mathrm{mg} / \mathrm{dl})$ & $50,4 \pm 16,5$ & $57,5 \pm 11,8^{a}$ & $44,2 \pm 7,1$ & $50,4 \pm 6,7^{\mathrm{a}}$ \\
\hline LDL-C(mg/dl) & $88,3 \pm 23,6$ & $77,5 \pm 21,7^{b}$ & $79,3 \pm 20,4$ & $69,0 \pm 20,9^{a}$ \\
\hline $\mathrm{TG}(\mathrm{mg} / \mathrm{dl})$ & $85,9 \pm 27,1$ & $74,6 \pm 25,3^{b}$ & $86,6 \pm 22,5$ & $77,2 \pm 27,9^{\mathrm{a}}$ \\
\hline $\operatorname{ApoA}(\mathrm{mg} / \mathrm{dl})$ & $128,8 \pm 20,9$ & $125,9 \pm 17,8$ & $126,0 \pm 17,4$ & $120,8 \pm 15,9$ \\
\hline LDL-C/HDL-C & $1,6 \pm 0,7$ & $1,5 \pm 0,5$ & $1,6 \pm 0,6$ & $1,5 \pm 0,5$ \\
\hline ApoB/ApoA & $0,4 \pm 0,1$ & $0,3 \pm 0,1^{b}$ & $0,4 \pm 0,1$ & $0,3 \pm 0,1^{b}$ \\
\hline
\end{tabular}

CT: colesterol total; HDL-C: lipoproteína de atta densidade; LDL-C: lipoproteína de baixa densidade; TG: triglicerídeos; ApoA: apolipoproteína Al; ApoB: apolipoproteína BI $00 ;$ a: $p<0,05 ;$ b: 0,00 < $<<0,05$

Tabela 2 - Odds ratio e intervalos de confiança de $95 \%$ para a associação entre o uso de tabaco e o perfil aterogênico de lipídeos-lipoproteínas plasmáticas em adolescentes

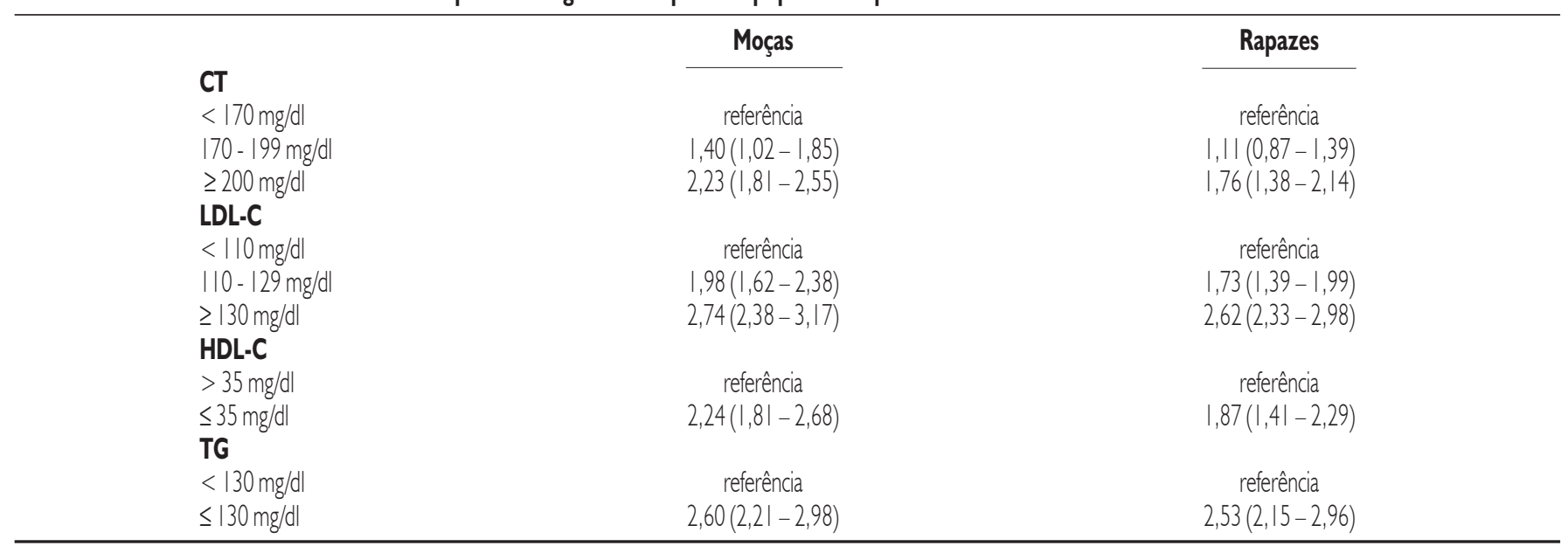

Os resultados equivalentes à análise de covariância, controlando estatisticamente os escores da RISCC, são mostrados na Tabela I. Os resultados apontam valores médios estatisticamente mais elevados entre as moças e os rapazes fumantes na maioria dos componentes plasmáticos considerados, independente dos escores da RISCC. As concentrações médias de ApoA e as relações CT/HDL-C, LDL-C/ HDL-C mostram-se semelhantes entre os fumantes e os não fumantes. Ao recorrer aos valores de referência sugeridos pela Sociedade Brasileira de Cardiologia 21 , verifica-se que um perfil lipídicolipoprotéico de risco aterogênico foi comum em 19\% das moças fumantes e 16\% dos rapazes fumantes. Entre os não fumantes, comprometimento lipídico-lipoprotéico foi identificado em II\% e $8 \%$ das moças e dos rapazes, respectivamente.

A extensão a que o uso de tabaco possa estar associado no que diz respeito aos níveis comprometedores de lipídeos-lipoproteínas plasmáticas foi estimada mediante análise de regressão logística binária. As dimensões de OR provenientes dos cálculos estatísticos são apresentadas na Tabela 2. Na presente casuística, aquelas moças fumantes demonstram possuir entre duas e três vezes mais chances de apresentarem perfil aterogênico de lipídeos- lipoproteínas plasmáticas. No caso dos rapazes, as dimensões encontradas de OR reduziram-se discretamente; contudo, ainda assim, permaneceram significativas em linguagem estatística.

\section{Discussão}

Embora o uso de tabaco seja considerado um dos mais importantes fatores de risco predisponentes ao aparecimento e ao desenvolvimento da aterosclerose $e^{5,7,19,20}$, os mecanismos agressores envolvidos com esse comportamento não estão totalmente elucidados. No entanto, algumas ações reversíveis quanto à exposição ao uso de tabaco têm sido documentadas experimentalmente em adultos: a indução a arritmias cardíacas por aumento da excitabilidade do miocárdio e maior liberação de catecolaminas, o aumento da concentração de monóxido de carbono na corrente sangüínea danificando o endotélio, a formação de carboxihemoglobina que desencadeia anoxemia nos tecidos, incluindo o miocárdio, e o aumento da agregação plaquetária ${ }^{27-29}$.

A minimização ou a remoção dessas ações deletérias podem explicar os imediatos benefícios à saúde cardiovascular em conseqüência do abandono do uso de tabaco. Também, os adultos fumantes tendem a apresentar lesões ateroscleróticas mais freqüentemente e 


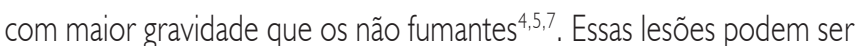
parcialmente explicadas pelas alterações observadas no perfil lipídicolipoprotéico plasmático dos fumantes.

A semelhança do que foi encontrado em outros estudos envolvendo populações jovens ${ }^{30,31}$, evidências reunidas no presente estudo apontam forte impacto dos componentes do tabaco na presença de um perfil aterogênico de lipídeos-lipoproteínas plasmáticas em adolescentes. Ao analisar a extensão da associação entre o uso de tabaco e 0 comprometimento nos níveis plasmáticos de CT, HDL-C, LDL-C e TG, verificou-se que, na maioria das situações, as dimensões das OR foram superiores a duas unidades. Sendo assim, na presente casuística, ser um adolescente fumante implica em um aumento de, no mínimo, duas vezes na probabilidade de se encontrar valores sugestivos de risco elevado quanto à presença de dislipoproteinemias. É importante destacar que, no delineamento do presente estudo, os possíveis efeitos de confusão associados à ingestão de gordura saturada e de colesterol dietético foram controlados mediante procedimentos estatísticos.

Estudos disponibilizados na literatura apontam que possíveis mecanismos que podem favorecer a presença de perfil aterogênico de lipídeos-lipoproteínas plasmáticas em fumantes são decorrentes da maior secreção de substâncias oxidativas pelas células da parede arterial induzida pela presença de nicotina e de monóxido de carbono na corrente sangüínea. Conseqüentemente, a capacidade de oxidação dos lipídeos circulantes tende a ser potencializada, elevando, por sua vez, as concentrações plasmáticas. Ainda, em resposta ao processo mais intenso de oxidação, poderá ocorrer maior acúmulo de éster de colesterol em macrófagos, precipitando, desse modo, a formação de células espumosas e de placas ateroscleróticas ${ }^{32}$.

Apesar das dosagens de TG, CT e suas frações serem importantes indicadores nas intervenções de controle dos fatores de risco predisponentes às coronariopatias, as quantidades de ApoA, principal proteína encontrada no HDL-C, e de ApoB, principal proteína associada ao LDL-C, são apontadas como melhores marcadores das lipoproteínas aterogênicas, fornecendo estimativas mais confiáveis e seguras quanto ao prognóstico de eventual aparecimento e progressão de lesões ateroscleróticas ${ }^{33,34}$. No presente estudo, os valores médios de ApoA observados nos adolescentes fumantes e não fumantes não apresentaram diferenças estatisticamente significantes. No entanto, os valores médios de $A p o B$ e da relação $A p o B / A p o A$ foram significativamente mais elevados entre as moças e os rapazes fumantes. Estudos disponibilizados na literatura sugerem que, em jovens fortemente predisponentes a apresentar sintomas associados às coronariopatias, são identificados níveis perniciosos de apolipoproteínas, sobretudo no que se refere à relação $A p o B / A p o A^{35,36}$.

$O$ conceito de que a aterosclerose é um processo degenerativo que pode se iniciar em idades jovens e avançar por vários estágios, evoluindo para uma posterior coronariopatia prematura na idade adulta, tem apontado para a necessidade de ações intervencionistas de prevenção já na adolescência. A quase totalidade dos adultos fumantes inicia o uso de tabaco antes dos 18 anos de idade ${ }^{10}$. Em nosso estudo, 97\% dos adolescentes analisados relataram ter iniciado o uso de tabaco antes dos 16 anos. Esses achados sugerem que os eventuais fatores ambientais, socioculturais e familiares com relação à aquisição de dependência quanto ao uso de tabaco exercem sua influência em idades muito precoces.
Neste particular, as condições predisponentes que podem levar os adolescentes a estabelecerem contato com o tabaco têm sido objeto de exaustivos estudos epidemiológicos. Evidências apontam que 0 hábito de fumar dos amigos e dos irmãos com mais idade está fortemente associado ao tabagismo. Baixo rendimento escolar, abandono dos estudos e trabalho remunerado também mostram estar associados com tabagismo. Desajuste familiar, separação dos pais e moradia com outras pessoas que não os pais biológicos são condições que aumentam a possibilidade do uso de tabaco ${ }^{37-41}$. Ingestão de bebidas alcoólicas é um comportamento intensamente relacionado com o tabagismo ${ }^{41}$. Nível socioeconômico e hábito de fumar dos pais são achados controversos; contudo, recursos financeiros disponíveis aos adolescentes tendem a influenciar no uso de tabaco ${ }^{42}$. Ainda, anúncios e promoção do tabaco veiculados nos meios de comunicação aumentam a probabilidade dos adolescentes começarem a fumar ${ }^{43}$.

\section{Conclusão}

Concluindo, chama-se a atenção para o fato de que os programas direcionados à adoção de um estilo de vida saudável, incluindo abstenção do uso de tabaco, deverão iniciar-se na adolescência na tentativa de prevenir ou retardar o desenvolvimento de lesões ateroscleróticas e minimizar o aparecimento de coronariopatias prematuras na idade adulta.

\section{Conflito de interesse: não há.}

\section{SUMMARY}

\section{TOBACCO USE AND PLASMA LIPID-LIPOPROTEIN PROFILE IN ADOLESCENTS}

OBJECTIVE. To analyze the impact of tobacco use on plasma lipid lipoprotein profile in representative sample of adolescents.

METHODS. A sample of 452 subjects (246 girls and 206 boys) I 5 to 18 years old were included in the study. Each participant completed a structured and self-administered questionnaire concerning tobacco use. Plasma lipid-lipoprotein concentrations were measured by standard procedures. Differences between mean values were evaluated by analysis of covariance, controlling for saturated fat and cholesterol intake. Odds ratio was used to estimate the relative risk of the smokers being classified with an undesirable level of a plasma lipidlipoprotein parameter.

RESULTS. The proportion ofsmokers was $20.9 \%$ for boys and $15.4 \%$ for girls. The average consumption of cigarettes perday was $9.2 \pm 4.7$ for boys and $5.6 \pm 3.1$ for girls. When compared with non-smokers, boy and girl smokers showed a significantly higher serum levels of total cholesterol, LDL-cholesterol, triglycerides and apolipoprotein B 100, and significantly lowerserumlevels of HDL-cholesterol. Adolescentsmokerstended to show atwo-fold higher risk ofaltered lipid-lipoprotein levels than non-smokers.

CONCLUSION. The present data could imply that intervention promoting a healthy lifestyle, including non smoking, should start at an early age to prevent or delay development of atherosclerotic lesions and ultimately to minimize the appearance of premature coronary heart disease in adults.[Rev Assoc Med Bras 2007; 53(I): 59-63]

KEY wORDS: Smoking. Apolipoprotein. Cholesterol. Adolescence. 


\section{REFERÊNCIAS}

I. US Department of Health and Human Services. Smoking-attributable mortality and years of potential life lost - Unites States, 1991. MMWR Morta Recomm Rep. 1998;40:62-71.

2. Peto R, Lopez A, Borham J, Thun M, Health C. Mortality from tobacco in developed countries: indirect estimation from national vital statistics. Lancet. 1992;339: 1268-78.

3. Prescott E, Scharling H, Osler M, SChnohr P. Importance of light smoking and inhalation habits on risk of myocardial infarction and all cause mortality. A 22 year follows up of I 2. 149 men and women in The Copenhagen City Heart Study. J Epidemiol Community Health. 2002;56:702-6.

4. Price JF, Mowbray PI, Lee AJ, Rumley A, Lowe GD, Fowkes FG. Smoking and cardiovascular risk factors in the development of cardiovascular disease and coronary artery disease: Edinburg Artery Study. Eur Heart J. 1999;20:344-53.

5. McBride PE. The health consequences of smoking: cardiovascular disease. Med Clin North Am. 1992;76:333-53.

6. Prescott E, Hippe M, Schnohr P, Hein HO, Vestbo J. Smoking and risk of myocardial infarction in women and men: longitudinal population study. BJM. 1998;316:1043-7.

7. Burns DM. Tobacco-related diseases. Semin Oncol Nurs. 2003; 19:244-9.

8. Sitas F, Urban M, Bradshaw D, Kielkowski D, Bah S, Peto R. Tobacco attributable deaths in South Africa. Tob Control. 2004: 13:396-9.

9. Mackay J, Eriksen MP. The tobacco atlas. Geneva: World Health Organization; 2002.

10. Warren CW, Riley L, Asma S, Eriksen MP, Green L, Blanton C et al. Tabacco use by youth: a surveillance report from the Global Youth Tobacco Survey Project. Bulletin World Health Organ. 2000;78:868-76.

I I. Murray CGL, Lopez AD. Alternative projections of mortality and disease by cause, 1990-2020: global burden of disease study. Lancet. 1997;349: | 498-504.

12. Global Youth Tobacco Survey Collaborative Group. Tobacco use among youth: a cross country comparison. Tob Control. 2002; I I:252-70.

13. Godeau E, Rahav G, Hublet A. Tobacco smoking. In: Currie C, Roberts C, Morgan A, Smith R, Settertobulte W, Samdal O, et al. editors. Health Behaviour in School-Aged Children (HBSC) Study: International Report from the 2001/2002 Survey. Copenhagen: WHO Regional Office for Europe; 2004. p.63-72.

14. Galduróz JCF, Noto AR, Fonseca AM, Carlini EA. V levantamento nacional sobre o consumo de drogas psicotrópicas entre estudantes do ensino fundamental e médio da rede pública de ensino nas 27 capitais brasileiras: 2004. São Paulo: CLR Balieiro Editores; 2005

15. Tovar GVJ, Lopes AFJ, Rodríguez SN. Recent trends in mortality due to chronic obstructive pulmonary disease (COPD) in México, 1980-2002. Arch Med Res. 2005;36:65-9.

16. Jindal SK, Gupta D. The relationship between tobacco smoke and bronchial asthma. Indian J Med Res. 2004; I 20:443-53.

17. Vineis $P$, Airoldi L, Veglia $P$, Olgiati L, Pastorelli $R$, Autrup $H$, et al. Environmental tobacco smoke and risk of respiratory cancer and chronic obstructive pulmonary disease smokers and never smokers in EPIC prospective study. BMJ. 2005;330:277.

18. Harris M. A smoking related triad: PAD, COPD and CCF. Aust Fam Physician. 2004;33:207- 10

19. Kamholz SL. Pulmonary and cardiovascular consequences of smoking. Med Clin North Am. 2004;88: I 4 5-30.

20. Kannel WB, Higgins M Smoking and hypertension as predictors of cardiovascular risk in population studies. J Hypertens. 1990;8:S3-S8.

21. ANEP. Critério de Classificação Econômica Brasil. São Paulo: Associação Nacional de Empresas de Pesquisa; 2000.

22. Tanner JM. Growth at adolescence. $2^{\text {nd }}$ ed. Oxford: Blackwell Scientific Publications; 1962.

23. Friedewald WT, Levy RI, Frederickson DS. Estimation of the concentration of low density lipoprotein cholesterol in plasma without use of the preparative ultracentrifuge. Clin Chem. 1972; 18:499-502.

24. Sociedade Brasileira de Cardiologia. III Diretrizes Brasileiras sobre Dislipidemias e Diretrizes de Prevenção da Aterosclerose do
Departamento de Aterosclerose da Sociedade Brasileira de Cardiologia. Arq Bras Cardiol. 200 I;77(Supl 3).

25. Guedes DP, Guedes JERP, Barbosa DS, Oliveira JA, Stanganelli LCR. Fatores de risco cardiovasculares em adolescentes: indicadores biológicos e comportamentais. Arq Bras Cardiol. 2006;86:439-50.

26. Anção MS, Cuppari L, Tudisco LS, Draibe AS, Sigulen D. Sistema de apoio à decisão em nutrição: Versão 2.5. Centro de Informática em Saúde da Universidade Federal de São Paulo. São Paulo: Escola Paulista de Medicina; 1993.

27. Winniford MD, Wheelan KR, Kremers MS, Ugolini V, Van den Berg E. Smoking-induced coronary vasoconstriction in patients with atherosclerotic coronary artery disease: evidence for adrenergically mediated alterations in coronary artery tone. Circulation. 1986;73:662-7.

28. Astrup P, Kjeldsen K. Model studies linking carbon mon-oxide and/or nicotine to arteriosclerosis and cardiovascular disease. Prev Med. 1979;8:295-302.

29. McGill HJr. Potential mechanisms for the augmentation of atherosclerosis and atherosclerotic disease by cigarette smoking. Prev Med. 1979;8:309403.

30. Craig WY, Palomaki GE, Johnson M, Haddow JE. Cigarette smokingassociated changes in blood lipid and lipoprotein levels in the 8-to-19year-old age group: a meta-analysis. Pediatrics. 1990; 85: I 5-8.

3I. Raftopoulos C, Bermingham MA, Steinbeck KS. Coronary heart disease risk factors in male adolescents, with particular reference to smoking and blood lipids. J Adolesc Health. 1999;25:68-74.

32. McGill HC Jr, McMahan CA, Malcom GT. Effects of serum lipoproteins and smoking on atherosclerosis in young men and women. Arterioscler Thromb Vasc Biol. 1997; 17:95- 106.

33. Grundy SM. Low-density lipoprotein, non-high-density lipoprotein, and apolipoprotein B as targets of lipid-lowering therapy. Circulation. 2002; 106:2526-9.

34. Jiang R, Schulze MB, Li T, Rifai N, Stampfer MJ, Rimm EB et al. Non-HDL cholesterol and apolipoprotein B predict cardiovascular disease events among men with type 2 diabetes. Diabetes Care. 2004;27: 1991-7.

35. Perova N, Aingorn H, Metelskaya T, Dorofeeva T, Belokonj N. Plasma lipid and apolipoprotein levels in children hereditarily predisposed to coronary heart disease. Acta Paediatr Scand. 1988;77:559-62.

36. Freedman DS, Srinivasan SR, Shear CL, Franklin FA, Weber LS, Berenson GS. The relation of apolipoproteins A-I and B in children to parental myocardial infarction. N Engl J Med. 1986;3 15:721-6.

37. Malcon MC, Menezes AMB, Chatkin M. Prevalência e fatores de risco para tabagismo em adolescentes. Rev Saúde Pública. 2003;37: I-7.

38. Burt RD, Dinh KT, Peterson AV Jr, Sarason IG. Predicting adolescent smoking: a prospective study of personality variables. Prev Med. 2000;30: 134-45.

39. Ariza C, Nebof M. Factors associated with smoking progression among spanish adolescents. Health Educ Res. 2002; 17:750-60.

40. Simantov E, Schoen C, Klein JD. Health compromising behaviors: why do adolescents smoke or drink? Identifying underlying risk and protective factors. Arch Pediatr Adolesc Med. 2000; I 54: I 025-33.

4I. Carvajal S, Wiatrek DE, Evans RI, Knee CR, Nash SG. Psychosocial determinants of the onsent of escalation of smoking: cross sectional and prospective findings in multiethnic middle school samples. J Adolesc Health. 2000;27:255-65.

42. Hidalgo AC, González B, Pinilla J, Barber P. Factores predoctores del inicio y consolidación del consumo de tabaco en adolescentes. Gac Sanit. 2005; 19:440-7.

43. Pierce J. Tobacco industry promotion on cigarettes and adolescent smoking. JAMA. 1998;279:5 | |-5. 